\title{
THE DYNAMICAL EVOLUTION OF BINARIES IN CLUSTERS
}

\author{
D. C. HEGGIE
}

Trinity College, and Institute of Astronomy, Cambridge, U.K.

\begin{abstract}
Using information on the rates at which binaries suffer encounters in a stellar system (Heggie, 1974a), we here study the effects of such processes on the evolution of the system itself. First considering systems with no binaries initially, we show that low-energy pairs attain a quasi-equilibrium distribution comparatively quickly. Their effect on the evolution of the cluster is negligible compared with that of two-body relaxation. In small systems energetic pairs may form sufficiently quickly to exercise a substantial effect on its development and on the escape rate, but in large systems their appearance is delayed until the evolution of the core is well advanced. In that case they appear to be responsible for arresting the collapse of the core at some stage.

Binaries of low energy, even if present initially in large numbers, are likely to have at most only a temporary effect on the evolution of the system. High-energy pairs are not easily destroyed, and so, if present initially, their effect is persistent. It competes with two-body relaxation especially when the fraction of such pairs and the total number-density are high, as in the core, where, in addition, binaries tend to congregate by mass segregation. When encounters with binaries become important, being mostly 'superelastic' they enhance escape and lead to ejection of mass from the core into the halo, thus accelerating the rate at which mass is lost by tidal forces. It is difficult to decide observationally whether globular clusters possess sufficiently large numbers of binaries for these effects to be important.
\end{abstract}

\section{Introduction}

Results from explicit computations of small $N$-body systems with up to 500 members (von Hoerner, 1963; van Albada, 1968; Aarseth, 1971a; Heggie, 1974a) have consistently revealed that phenomena involving the dynamical formation and development of binary stars play some role in the evolution of clusters of stars. On the other hand, the study of much larger collisional systems is at present practicable only if use is made of rapid approximate methods based on the Fokker-Planck equation (Larson, 1970a, b; Hénon, 1971; Spitzer and Hart, 1971a, etc.). Since such methods implicitly ignore three-body encounters, which are essential for the binary phenomena whose effects in explicit $N$-body calculations are so striking, it has become a matter of some importance to establish whether or not the binaries themselves have a significant influence on the overall evolution of the cluster, or whether, on the contrary, they form and develop automatically as a by-product in response to the evolution of the cluster by the usual relaxation processes in which binaries do not essentially participate.

Although it seems almost paradoxical, the simplified methods, which ignore threebody interactions, agree in predicting that three-body phenomena become important; for, as Hénon (1971) noted, the effect of collisional relaxation, whereby an outward flux of mass accompanies an inward flux of binding energy, leads to conditions favourable for the formation of one or more energetic binaries. Therefore he supposed that the binaries observed to form in direct $N$-body calculations were a symptom of this phenomenon, and thus a consequence of evolution of the cluster rather than a cause. Spitzer and Hart (1971a) improved the argument by outlining some 
facts on the formation and evolution of binaries in clusters. If we define 'soft' binaries to be those with binding energies $x$ satisfying $\beta x<1$, where $\frac{3}{2} \beta^{-1}$ is the local mean kinetic energy of the single stars, they remarked that the effect of such pairs was negligible because their total binding energy must be small and, furthermore, they must exhibit a tendency to be disrupted. For 'hard' binaries, which by definition satisfy the inequality $\beta x>1$, the argument differs, for these may absorb binding energy, but Spitzer and Hart showed, by a calculation to orders of magnitude, that the number of such pairs forming per unit relaxation time must vary approximately as $N^{-1}$, where $N$ is the total number of particles in the system. Since the evolution of the cluster will be over after at most a hundred relaxation times, the conclusion is that the formation of hard pairs can be entirely ignored, provided that the system is of sufficient size.

Since considerable theoretical effort has been applied to a detailed, independent investigation of dynamical processes involving binaries, the rate of their occurrence can now be stated with some accuracy in most cases (Heggie, 1974a). In this paper these newly available results are applied to a re-examination of the role played by binaries in the evolution of clusters, with especial regard to the questions discussed by Spitzer and Hart. In the next two sections, devoted respectively to soft and hard pairs, the arguments will be seen to differ in some respects from those quoted above, although the broad conclusions of Spitzer and Hart are preserved. In particular it is confirmed that in a large system there is insufficient time for the formation of enough binaries to affect significantly the evolution of the system. In the fourth section we consider how these conclusions must be modified if we do not wait for the binaries to form by these inefficient dynamical processes: supposing that there may already exist numbers of binaries when the star cluster has formed, we enquire how abundant and how energetic they need to be in order to exert a significant influence on the dynamical evolution of the whole cluster. Throughout most of the paper, except where otherwise stated, the discussion is restricted to systems whose members all have the same mass, $m$, and so the final section indicates certain avenues which require exploration before the results may be applied to real systems, with especial regard to the effects of a mass-spectrum. At this point some suggestions are made for contact with observations.

First, however, we remark on the assumptions and approximations made in order to arrive at the 'reaction rates' which we shall quote, though full details will be found elsewhere (Heggie, 1974a). The results are expressed in terms of rate functions $Q(x, y)$, analogous to those of atomic physics, defined thus:

Let $n$ be the number-density of single stars near a binary having 'internal' binding energy $x>0$. Then the probability that, during an encounter with a single star, the binary suffers a change in its binding energy lying in the range $(y, y+\mathrm{d} y)$ during the time interval $(t, t+\mathrm{d} t)$ is defined to be $n Q(x, y) \mathrm{d} y \mathrm{~d} t$.

This definition is easily extended to accommodate the case in which stars with different masses are present, but we note that the encounter responsible for a certain change in energy is idealised to occur instantaneously. Since only the local number- 
density enters, we consider solely binaries with semi-major axes much smaller than the local density scale height in the cluster. For the same reason the results are restricted to encounters with stars at impact parameters much less than this scale height; however, since the gravitational perturbation of a distant star on the internal motion of a binary is tidal, the total effect of the neglected very distant encounters is normally weak and negligible. It is assumed that, prior to the encounter, the distributions of the binary and the single star participating in it are uncorrelated, and that the distributions of the velocity of the single star and of the velocity of the masscentre of the binary are Maxwellian. The initial energy of the binary is fixed at $x$, and the orientation and the phase of its orbit are random. The distribution of its eccentricity, $e$, is assumed to be $f(e)=2 e$; there is substantial theoretical justification for this and it is well confirmed numerically (Heggie, 1974a; also examples in van Albada, 1968; and Aarseth and Hills, 1972). The results of other numerical experiments suggest that the analytically derived rates are correct within a factor of two, and often better than this.

Another useful preliminary is an expression for the relaxation time, for which we adopt the expression

$$
t_{r h}=\frac{0.0600}{\log (0.4 N)}\left(\frac{N R_{h}^{3}}{G m}\right)^{1 / 2}
$$

(Spitzer and Hart, 1971a), where logarithms to the base of ten are intended, and $R_{h}$ is the radius containing the innermost half of the mass. From the same source we have

$$
\frac{G N m^{2}}{R_{h}} \simeq 7.5\left\langle\beta^{-1}\right\rangle
$$

where the average value of any local quantity, $f$, is defined to be

$$
\langle f\rangle \equiv N^{-1} \int f n \mathrm{~d}^{3} \mathbf{r},
$$

the integral being taken over the volume of the cluster. These authors also define a dynamical time, closely related to the crossing time, by the equation

$$
t_{d h}=\frac{1.58 R_{h}^{3 / 2}}{(G N m)^{1 / 2}},
$$

and, finally, we introduce the quantity $n_{h}$, defined to be the mean number-density within a distance $R_{h}$ of the centre of the cluster, whence

$$
n_{h} \equiv \frac{3 N}{8 \pi R_{h}^{3}} \text {. }
$$




\section{Soft Binaries}

It can be shown by several methods (e.g. Gurevich and Levin, 1950) that, if pairs of stars in a system are uncorrelated, the space density of pairs with binding energies in the range $(x, x+\mathrm{d} x)$ is approximately $n_{0}(x) \mathrm{d} x$, where

$$
n_{0}(x)=\frac{1}{2} \pi^{3 / 2} n^{2} \beta^{3 / 2} G^{3} m^{6} x^{-5 / 2},
$$

provided that single stars have a Maxwellian distribution of velocities and

$$
N^{-1} \ll \beta x \ll 1
$$

This means that we consider only very soft pairs with separations much less than the typical density scale height, whence once again knowledge of only the local number-density will suffice. A rather descriptive argument (Heggie, 1974a) may be used to show that (4) will generally be the distribution of binding energies observed by soft pairs with energies in the range $6 N^{-1} \ll \beta x \ll 3 N^{-1 / 2}$, but the following more thorough discussion is applicable also to the range (5).

Now we may remark that $n_{0}(x)$ is approximately an equilibrium distribution in the range (5), provided that the cluster has evolved to an extent sufficient to ensure that the distribution of velocities is approximately Maxwellian. To demonstrate this we need the kinetic equation for $n(x)$, the absence of the subscript zero implying that we here do not necessarily suppose that the number-density of binaries has the form (4). It is

$$
\begin{aligned}
& \frac{1}{n} \frac{\partial n(x)}{\partial t}=n^{2} Q(x)-n(x) Q(x,-\infty)+ \\
& +\int_{0}^{\infty} \mathrm{d} x^{\prime}\left\{n\left(x^{\prime}\right) Q\left(x^{\prime}, x-x^{\prime}\right)-n(x) Q\left(x, x^{\prime}-x\right)\right\},
\end{aligned}
$$

where the new quantities $Q(x), Q(x,-\infty)$ are defined to be, respectively, the formation and destruction rates for pairs of energy $x$, in a manner akin to $Q(x, y)$.

It may be shown by general arguments that the rate functions $Q$ must satisfy the 'detailed balance' conditions (cf. Ross et al., 1969)

and

$$
n^{2} Q(x)=n_{0}(x) e^{\beta x} Q(x,-\infty)
$$

$$
n_{0}(x) e^{\beta x} Q\left(x, x^{\prime}-x\right)=n_{0}\left(x^{\prime}\right) e^{\beta x^{\prime}} Q\left(x^{\prime}, x-x^{\prime}\right) .
$$

Indeed, by consideration of the dynamics of individual encounters (Heggie, 1974a) one obtains approximate expressions for the rate functions, and these are actually found to satisfy the quoted relations within the accuracy of the approximations. One implication is that the distribution

$$
n(x)=n_{0}(x) e^{\beta x}
$$

is an equilibrium solution of (6), provided that $n$ and $\beta$ are constants. 
This distribution is physically unrealistic for $\beta x \rightarrow \infty$, but in the range specified by (5) it is approximately $n_{0}(x)$. Hence the distribution (4) is approximately an equilibrium distribution, provided that $n$ and $\beta$ are constants. Generally speaking, this will also be true approximately even if $(7)$ is not satisfied for $\beta x \gtrsim 1$, provided that changes in energy between values within the range (5) occur with far greater frequency than changes from energies within this range to energies in the range $\beta x \gtrsim 1$. That this is the case can be seen by examining the appropriate form (Heggie, 1974a)

$$
Q(x, y) \propto G^{2} m^{7 / 2} \beta^{1 / 2} y^{-2}\left(4 \frac{x}{y}+7\right)\left(\frac{x}{x+y}\right)^{5 / 2} \text { when }(\beta x)^{3 / 2} \ll \beta y,
$$

for the rate function: if $\beta x \ll 1$ the rate for energy changes $y$ satisfying $\beta y \simeq 1$ is much less than that for changes satisfying the inequality $\beta y \ll 1$. Hence we conclude that (4) is approximately an equilibrium distribution of binding energies in the range (5), if $n$ and $\beta$ are constants.

The initial conditions in most computational studies of $N$-body systems are such that there is no pair correlation to begin with, but in general binaries may not be present initially with the distribution (4) and, even if they are, the quantities $n$ and $\beta$ will change with time as a result of the familiar relaxation processes, and so the distribution will not remain in equilibrium for long. We therefore investigate whether deviations from equilibrium, however generated, tend to grow or to decline. Setting $n(x) \equiv n_{0}(x)+\delta n(x)$, we see that the deviation $\delta n(x)$ satisfies the same equation as $n(x)$, i.e. (6), except that the first ('creation') term on the right-hand side is absent. Now soft binaries are normally destroyed at a rate given by

$$
Q .(x,-\infty)=\frac{20}{3} \sqrt{\frac{\pi}{3}} G^{2} m^{7 / 2} \beta^{1 / 2} x^{-1}
$$

approximately, while the rate at which the energy of a soft pair changes as a result of encounters which do not destroy it is

$$
\dot{x} \simeq n G^{2} m^{7 / 2} \beta^{1 / 2}\left(-\frac{\pi}{2}+4 \sqrt{\frac{\pi}{3}}\left\{\frac{5}{3}-\ln \frac{4}{\beta x}\right\}\right)
$$

(Heggie, 1974a). When binaries are distributed according to (4), the formation of new pairs approximately balances the destruction of those present. In the absence of a creation mechanism, the number of soft pairs would diminish because of their destruction, and by (9) the mean energy of those surviving would decrease, thus accelerating the destruction process. Gurevich and Levin (1950) and Spitzer and Hart (1971a) showed that this tendency of soft pairs to be disrupted by encounters could be understood using an equipartition argument. We conclude that $\delta n$ tends to zero, i.e. the distribution of soft pairs tends to approximately $n_{0}(x)$ given by $(4)$, in consequence of three-body encounters.

The time scale for the approach of the distribution of soft pairs to equilibrium may 
be estimated from (1) and (8) as

$$
\begin{aligned}
t_{s} & =\frac{1}{n Q(x,-\infty)}, \\
& \simeq 1.00 \log (0.4 N) \beta x\left(n^{-1} n_{h}\right)\left(\beta\left\langle\beta^{-1}\right\rangle\right)^{-3 / 2} t_{r h} .
\end{aligned}
$$

As Gurevich and Levin in effect pointed out, a simple argument shows that $t_{s}$ must be much less than $t_{r h}$ for very soft pairs, for the latter is the time required on average for changes in energy of order $\beta^{-1}$, while the disruption of a soft pair requires only a much smaller change, of order $x$. By comparison, Larson's estimate for the time required for the complete evolution of a dense central core is at least $16 t_{r h}$ (cf. Spitzer and Hart, 1971b). We therefore expect that, even if soft pairs are not initially distributed according to (4), their distribution will take approximately this form after a time which is small compared with the time taken for what Spitzer and Hart term 'complete collapse' of the core. Then, as the parameters $n$ and $\beta$ vary because of the evolution of the system, soft pairs will remain approximately in equilibrium provided that such changes take place on a time scale which is long compared with $t_{s}$. If the time scale is of the order of $t_{r h}$, this will certainly be true for very soft pairs, and their distribution will change on a time scale which is entirely governed by that of the evolution of the single-particle distribution function. From now on we shall assume that soft pairs satisfying the inequality (5) will be distributed in accordance with (4).

Using this distribution, the total number and the total binding energy of such binaries are found to be of the order of

and

$$
90 N^{1 / 2}\left\langle n \beta^{3}\right\rangle\left\langle\beta^{-1}\right\rangle^{3} n_{h}^{-1}
$$

$$
200 N^{-3 / 2} E\left\langle n \beta^{2}\right\rangle\left\langle\beta^{-1}\right\rangle^{2} n_{h}^{-1}
$$

respectively, where $E$ is the total energy of the system. The total number of such pairs and, even more so, the total binding energy, both become relatively negligibly small for a sufficiently large system. For example, in the case of Plummer's model, $\left\langle n \beta^{3}\right\rangle\left\langle\beta^{-1}\right\rangle^{3} n_{h}^{-1} \simeq 0.36$, although (4), (11) and (12) all require some adjustment of coefficients for this model (Heggie, 1974a) because of the non-Maxwellian distribution of velocities.

There is another reason why the existence of very soft pairs poses no threat to the validity of fast methods for studying the evolution of clusters on the basis of twobody encounters. In such systems pairs of stars are uncorrelated except during hyperbolic encounters, and we recall that the equilibrium distribution of bound soft pairs (4) corresponds to an absence of correlation in the two-body distribution function. Therefore these same fast methods correctly, if implicitly, describe the equilibrium distribution to which we expect very soft pairs will adhere throughout most of the lifetime of the system. Finally, since the soft pairs generally exist in the absence of correlations, there is no justification for regarding them as donating a separate contribution to the binding energy of the system. 


\section{Hard Binaries}

The destruction of very hard pairs of energy $x$ takes place on a time scale $t_{h}$ which is defined by analogy with $t_{s}$ in (10) and has the approximate value

$$
t_{h} \simeq 0.7 \log (0.4 N)\left(n^{-1} n_{h}\right)\left(\beta\left\langle\beta^{-1}\right\rangle\right)^{-3 / 2}(\beta x)^{2} e^{\beta x} t_{r h} .
$$

It will be noted that the values quoted for $t_{s}$ and $t_{h}$ are very comparable when $\beta x \simeq 1$, as indeed they should be, and that $t_{h}$ is approximately equal to the quoted estimate for the time of complete core evolution for a certain value of $\beta x$ which is fairly insensitive to the values of $n$ and $\beta$. In case $N=10^{5}$ and $\beta\left\langle\beta^{-1}\right\rangle=1$, some representative values are given in Table I. For substantially more energetic pairs, the proba-

\section{TABLE I}

Values of $\beta x$ at which $t_{h} \simeq 16 t_{r h}$

\begin{tabular}{rl}
\hline$n / n_{h}$ & $\beta x$ \\
\hline 1 & 1.2 \\
10 & 2.3 \\
100 & 3.6 \\
\hline
\end{tabular}

bility of direct disruption may be ignored but, as in the case of soft pairs, we must enquire whether 'cascades' (i.e. successions of changes in binding energy less than that required for disruption) act so as to increase or to decrease the lifetime of a hard pair.

For this and other purposes we require the following approximate expressions for the rate functions for hard pairs (Heggie, 1974a): from 'close' encounters

$$
Q(x, y) \simeq \begin{cases}45 G^{2} m^{7 / 2} \beta^{1 / 2} x^{-2} e^{\beta y} & (y<0) \\ 45 G^{2} m^{7 / 2} \beta^{1 / 2} x^{5 / 2}(x+y)^{-9 / 2}, & (y>0)\end{cases}
$$

and from 'distant' encounters

$$
\begin{aligned}
& Q(x, y) \simeq\left\{\begin{array}{c}
4.9 G^{2} m^{7 / 2} \beta^{1 / 2} x^{-1}|y|^{-1}\left|\ln \frac{|y|}{x}\right|^{-1 / 3} e^{\beta y} \\
(-\beta x \ll \beta y \ll-1) \\
2.4 G^{2} m^{7 / 2} \beta^{1 / 2} x^{-1}|y|^{-1}\left|\ln \frac{|y|}{x}\right|^{-1 / 3} \\
\left(\beta y_{0} \ll|\beta y| \ll 1\right) \\
4.9 G^{2} m^{7 / 2} \beta^{1 / 2} x^{-1} y^{-1}\left|\ln \frac{y}{x}\right|^{-1 / 3},
\end{array}\right. \\
& (1 \ll \beta y \ll \beta x)
\end{aligned}
$$

where $\beta y_{0}$ is a certain very small positive number. In order to evaluate the average rate of change, the ranges over which the latter set of three expressions are held to 
be valid must be extended slightly so as to be contiguous. Thus the boundary between (16) and (17) is taken to lie at $\beta y=-1$, and (16) and (18) are taken to hold only when

$$
|\beta y|<A \beta x,
$$

where $A$ is some number less than unity. Hence from close encounters we obtain

$$
\dot{x} \simeq 0.8\left(n n_{h}^{-1}\right)\left(\beta\left\langle\beta^{-1}\right\rangle\right)^{3 / 2} \frac{1}{\log (0.4 N)} \beta^{-1} t_{r h}^{-1} \quad(\beta x \gg 1) .
$$

From distant encounters the contribution is of the same functional form, but the coefficient is about 1.0 when $A=1$ and roughly proportional to $A$. The result that the rate of change of binding energy is independent of $x$ may seem surprising, but it can be understood easily from a simple argument (Heggie, 1974b).

The result (20), together with the similar result for distant encounters, implies that hard pairs which escape disruption rapidly become harder, thus strongly reducing the probability of disruption. In fact from (20) we may obtain an upper limit for the time scale over which a hard pair increases its energy by an amount of order $\beta^{-1}$, and Table I implies that during this time the disruption probability decreases by a factor of about ten. Hence a more accurate estimate of the binding energy at which disruption becomes unimportant may be obtained by equating this time scale with $t_{h}$, whence the critical value of the binding energy is given by $\beta x \simeq 0.9$. Many of the quoted expressions are inaccurate for such low values of $\beta x$, but we are probably safe in neglecting the disruption of pairs with energies exceeding $B \beta^{-1}$, where $B$ is some number around two.

The total rate at which such pairs form, including those created by cascade from the population of soft binaries distributed according to (4), is approximately

$$
\frac{300}{\log (0.4 N)} \frac{\left\langle\beta^{9 / 2} n^{2}\right\rangle}{\left\langle\beta^{-1}\right\rangle^{-9 / 2} n_{h}^{2}} B^{-7 / 2} N^{-1} t_{r h}^{-1},
$$

where again we have been obliged to extend the ranges of validity of some of our formulae; for example it is assumed that (4) holds for $N^{-1} \ll \beta x \lesssim 1$. The average energy of these new binaries is about

$$
\frac{7}{5} B\left\langle\beta^{-1}\right\rangle \frac{\left\langle\beta^{7 / 2} n^{2}\right\rangle}{\left\langle\beta^{-1}\right\rangle\left\langle\beta^{9 / 2} n^{2}\right\rangle} .
$$

From (21), using the values of the averages for a uniform sphere, we expect to obtain approximately

$$
\frac{4.8 \times 10^{3}}{N \log (0.4 N)} B^{-7 / 2}
$$

hard pairs after the lapse of about $16 t_{r h}$. In the case of Plummer's model the corresponding constant is about $1.5 \times 10^{3}$. Hence, taking $B \simeq 2$ in the case of a uniform sphere, at least one hard pair can form and survive within the time required for col- 
lapse of the core only if the total number of particles is less than about 200, although such results are rather sensitive to the chosen value of $B$. At any rate, in such a case a hard binary could, according to (20), absorb only about $2 \%$ of the total energy of the system, which is $\frac{3}{2} N\left\langle\beta^{-1}\right\rangle$, in the lifetime of the core, and the initial energy of the pair would be typically rather modest, i.e. $\simeq 3\left\langle\beta^{-1}\right\rangle$ on average.

For smaller systems the number of pairs expected, and the fraction of the energy of the system which they might be expected to absorb, should both increase. On the other hand, the dependence of the formation rate (21) on $N^{-1}$, which was obtained by Spitzer and Hart (1971a) using a much simpler argument, ensures that hard pairs will not normally have time to form in systems containing more than several hundred members. Furthermore, even if a hard pair were to form, the energy which it could absorb within the time required for complete collapse of the core would not be a significant fraction of the total energy of the system. To sufficiently large systems, therefore, fast methods of studying evolution which implicitly ignore processes involving binary stars may, in this respect at least, be applied with confidence. Direct comparison between the Monte Carlo method and explicit $\mathrm{N}$-body integrations (Hénon, 1971; Aarseth et al., 1974) provides complementary empirical support for the conclusion that this assumption made in the former method is approximately valid, certainly as far as early evolution of the cluster is concerned.

One qualification remains to be raised, for the computational evidence on systems with $N=250$ or 500 and a spectrum of masses seems at first sight to be in contradiction with the predictions given above, in that a large fraction of the total energy comes to reside in hard pairs in a time comparable with that required for complete collapse of the core (Aarseth, 1971a). Admittedly the evidence from the equal-mass models is less clear-cut (Aarseth, 1974) and it is for this case that the above theory has been developed, but in any event there is one aspect of the theory which we have ignored so far.

The factor $\left\langle\beta^{9 / 2} n^{2}\right\rangle$ in (21) is changing with time as a result of the usual relaxation mechanisms, which have nothing to do with the evolution of binaries, and possibly in addition as a result of the development of binaries. Late in the evolution of the core of a stellar system the number-density at a small distance, $r$, from its centre takes a form which has been quoted for small $N$ as

$$
n \propto r^{-12 / 5}
$$

(von Hoerner, 1968), while Larson (1970b) obtains a steeper profile, with the logarithmic density gradient around -3 or -4 , in a region which extends ever closer to the centre as time proceeds. The variation of $\beta$ with $r$ is much less steep, and so the quantity $\left\langle\beta^{9 / 2} n^{2}\right\rangle$ is increasing with time as evolution of the core draws towards its close, and by (21) the rate at which hard binaries form should increase proportionately. Alternatively one might regard the core crudely as an independently evolving $N$-body system with a decreasing number of members; when its membership is sufficiently small, (21) shows that hard binaries will form and evolve at a rate large enough to be important. 
At this stage it becomes of interest to consider how the number of binaries formed during the whole collapse of the core depends on $N$. For this purpose we shall assume that the central number-density and mean kinetic energy vary asymptotically with time approximately as

$$
n_{c}=n_{0}\left(\frac{\tau}{\tau_{0}}\right)^{m}, \quad \beta_{c}^{-1}=\beta_{0}^{-1}\left(\frac{\tau}{\tau_{0}}\right)^{n},
$$

where $m$ and $n$ are constants whose values, drawn from several sources, are given in Table II, $-\tau$ is the time measured in such a way that collapse of the core occurs as $\tau \rightarrow 0$, and a subscript zero denotes the initial value. Calculations show (Larson, $1970 \mathrm{a}, \mathrm{b})$ that, close to the centre of a cluster with a highly evolved core, there is a region of approximately uniform density, and we shall estimate its radius by treating this region as a cluster to which (2) is applicable with central values of density and 'temperature'.* Hence we find the number of stars within the central region to vary as

$$
N_{c}=N_{0}\left(\frac{\tau}{\tau_{0}}\right)^{-(m / 2)+(3 n / 2)},
$$

and its kinetic energy as

$$
E_{c}=E_{0}\left(\frac{\tau}{\tau_{0}}\right)^{-(m / 2)+(5 n / 2)} .
$$

Taking $\tau_{0}=16 t_{r h}$, we obtain from (21) the result that the total number of hard persistent pairs formed within the central region during the time $-\tau_{0}<-\tau<-\tau_{f}$ is approximately

$$
\begin{aligned}
& \frac{9.6 \times 10^{3} B^{-7 / 2}}{|6 n-3 m-2| \log (0.4 N)} N_{0} N^{-1}\left(n_{0} n_{h}^{-1}\right)^{2}\left(\beta_{0}\left\langle\beta^{-1}\right\rangle\right)^{9 / 2} \times \\
& \times\left\{\frac{N_{0}}{2 N}\left(N \frac{N_{0}}{2 N}\right)^{(3 n-2 m-2) /(3 n-m)}-\frac{1}{N}\right\},
\end{aligned}
$$

where $-\tau_{f}$ is taken to be the time at which the number of core members reaches the minimum value consistent with finite binding energy, i.e. two (cf. von Hoerner, 1968). When the density profile is as steep as, say, that given by (22), the number of binaries formed outside the core is at most comparable with the number formed inside it, which is given by (25).

The prediction of the actual numbers of binaries formed requires a careful choice of the initial conditions $\beta_{0}, n_{0}$ and $N_{0}$, which in turn depend on $N$, but, if we assume that such ratios as $N / N_{0}$ are independent of $N$, then the only factors which will vary

* The resulting exponents agree to within 0.04 of those obtained for the radius at which the density falls to $10^{-2}$ of its central value, as obtained from the small-scale figures in Larson's papers. The differences are systematic, however, in that the decrease of radius with time is slightly steeper when taken from the figures than when obtained via (2). 
with $N$ in the above formula are the logarithmic term in the first denominator, and the term within the bracket. Now we require values of the indices $m$ and $n$, and a list of those which have been obtained from theoretical or computational studies is given in Table II. The conclusion from the theoretical models is clear enough: from

TABLE II

Exponents for the evolution of the core

\begin{tabular}{llllll}
\hline Source & $m$ & $n$ & $\frac{3 n-2 m-2}{3 n-m}$ & $m-\frac{3}{2} n+1$ & $-\frac{5 n-m}{3 n-m}$ \\
\hline von Hoerner (1958) & & & & 0.64 \\
Miller and Parker (1964) & -1.56 & -0.37 & 0.02 & -0.01 & 0 \\
Spitzer and Saslaw (1966) & $-10 / 7$ & $-2 / 7$ & 0 & 0 & 0 \\
von Hoerner (1968) & $-4 / 3$ & $-2 / 9$ & 0 & 0 & $-1 / 3$ \\
Larson (1970a) & -1.34 & -0.225 & 0.01 & -0.00 & -0.32 \\
Larson (1970b) & -1.53 & -0.30 & 0.25 & -0.08 & -0.05 \\
Larson (1970b) & -1.54 & -0.30 & 0.28 & -0.09 & -0.06 \\
Larson (1970b) & -1.67 & -0.29 & 0.59 & -0.24 & -0.28 \\
Larson (1970b) & -1.47 & -0.30 & 0.07 & -0.02 & 0.05 \\
\hline
\end{tabular}

a Larson notes that the behaviour of $n_{c}$ and of $\beta_{c}^{-1}$ is not precisely represented by a power law, the indices changing slightly with $\tau$. The behaviour found by King (1958) is qualitatively similar in this respect, although $\tau$ cannot be defined in quite the same way because the particle number does not quite vanish after any finite time in this theory. However, these two effects are not related, for in King's theory it arises because of the variation with $N$ of the logarithmic term in the expression for the relaxation time, and in Larson's calculations this term is taken as a constant.

the third column of figures we see that the number of persistent hard binaries formed should be approximately independent of the number of particles. For some of Larson's models, however, many more pairs will form in larger systems as the core collapses than in small ones.

When such a binary forms in the core, it does so with an energy which is a few times the current value of the mean kinetic energy per particle. The binary then hardens at a rate given by (20), while $\beta_{c}$ is varying in accordance with (23). If $\beta_{c} x$ decreases with time, the binary effectively becomes softer, and it will ultimately be disrupted. On the other hand, if $\beta_{c} x$ increases with time, then the typical changes which its binding energy exhibits as a result of encounters with single stars will eventually become so much larger than $\beta_{c}^{-1}$, which must be of the order of the energy of escape from the core, that the binary will be ejected from the core. Only if $\beta_{c} x$ is approximately constant will the binary persist in the core as a hard pair. From (20) and (23) we readily find that

$$
\beta_{c} x \sim\left(\frac{\tau}{\tau_{0}}\right)^{m-(3 n / 2)+1}
$$

as $\tau \downarrow 0$, and the exponent here is given in the second last column of Table II. The prediction is, then, that in those cases where only a few binaries are expected to form, they will remain as hard pairs in the core; whereas if conditions are such that many 
pairs will form in large systems, then they will be continually ejected from the core, if not from the cluster. Such loss of mass by the core naturally tends to inhibit its further development if the flux is large enough.

It is unclear what will be the total energy of the binaries. If the energy of the core is taken as an upper limit, we see from (24) and the kind of argument used to arrive at (25) that the final energy of the core is roughly

$$
E_{0}\left(N_{0} / 2\right)^{-(5 n-m) /(3 n-m)} \text {. }
$$

The exponent here is given in the final column of Table II, from which we remark only that it is uncertain whether the fraction of energy absorbed by hard binaries increases or decreases as we proceed to a consideration of larger systems. For this and several other purposes it would be very helpful to possess a theory of core evolution by which the differences in the exponents found by Larson could be understood, as well as their relationship with the exponents predicted from previous theories.

At any rate, the conclusion must be that, in large systems, binaries evolve as a consequence of the development of the system by other processes, and it is only towards the very end of its evolution that binaries will appear. Therefore, fast methods which ignore the formation of binaries should be applicable throughout all except the very last stages of core evolution, and estimates of the time scale for the complete collapse of the core derived by such methods should be reliable to this extent.

Some confirmation of these conclusions emerges from a study of the results of published $N$-body experiments, if we compare the time scale over which hard binaries evolve with that for complete collapse of the core. In a small system the two should be comparable, while in a large system the evolution of binaries should occur rather suddenly after a lengthy period in which the system evolves in the absence of hard pairs. Somewhat striking evidence that this is so is provided by graphs showing the binding energies of hard pairs present at different times. When $N=10$ (van Albada, 1968), the energy of the hardest pair present increases fairly steadily, in marked contrast with the rather abrupt appearance and development of a hard pair in a much larger system $(N=500$ : Aarseth, 1971a), although one cannot easily rule out the effects of a different choice of mass-spectrum.

At this stage it is pertinent to remark on the mass-dependence of binary processes, a point which will be taken up again in the final section. First, the formation rate for pairs of a certain energy (Heggie, 1974a) increases strongly with increasing masses of the components, a fact that can be understood also by a phase-space volume argument (Miller, 1975). This is even true when the mass distribution is as steeply weighted against large masses as that used by Aarseth (1971b), viz $f(m) \propto m^{-2}$. Second, the number-density of the largest masses is especially high where binary formation is most rapid, i.e. at the centre of the cluster, because of the phenomenon of mass segregation, a fact which further favours the formation of binaries with components of high mass.

Once a binary has formed, its components may change identity only through 'ex- 
change' or 'resonance'. It has been noted (Heggie, 1974a) that it is unlikely for either component of a massive pair to be replaced by a particle of much smaller mass by exchange, while there is substantial numerical evidence (Anosova, 1969; Szebehely, 1972; Valtonen, 1974) that the least massive component of a bound triple system has much the highest probability of escape. Aarseth (1971a) has emphasised, in fact, that the final components of energetic pairs tend to be drawn from the two or three most massive particles even in the largest $N$-body systems studied.

Comparatively little attention has been paid either theoretically or computationally to the evolution of clusters after the development of the core has reached completion. Clearly the formation of new pairs will continue in the old halo, though at a rate much lower than that during the collapse of the core, and their energies are unlikely to be as great as those of the binaries which comprise the residue of the core. If there are many such pairs they may interact among themselves, leaving a few very energetic binaries, and thus resulting in a situation not unlike that which would have arisen had the evolution of the core led to the creation of only a small number of pairs in the first place.

Encounters with a hard pair of energy $x$ have several important effects on the single stars, and one of these we now discuss. If the encounter is sufficiently distant, the energy of the binary is almost unchanged and the encounter is 'elastic'. It merely contributes to two-body relaxation, the binary behaving like a single star whose mass equals the combined mass of its components, and the amount of energy exchanged is at most of order $\beta^{-1}$. If, however, the single star approaches to a distance sufficiently close that the change, $y$, in the binding energy of the binary considerably exceeds $\beta^{-1}$, the encounter is essentially 'superelastic', since $y>0$ in general. If $\varepsilon$ is the binding energy of the star to the cluster, then $\varepsilon$ suffers a change $\Delta \varepsilon \simeq-\frac{2}{3} y$. Since $y$ can be of order $x$, typically, a close encounter between a hard binary and a single star can lead to the escape of the star with high energy, and possibly also to the ejection of the binary. In fact there is direct evidence from computational studies of systems with $N \leqslant 500$ (van Albada, 1968; Aarseth, 1971a, 1974; Heggie, 1974a) linking hard binaries with escape at high energy.

In the presence of one hard pair of energy $x$, the escape rate is

$$
\mathscr{R}=n \int_{3 \varepsilon / 2}^{\infty} Q(x, y) \mathrm{d} y .
$$

Using (15) and (18) we readily obtain the result

$$
\mathscr{R} \simeq 4.1 \frac{n}{n_{h}}\left\langle\beta^{-1}\right\rangle^{3 / 2} \beta^{1 / 2} \varepsilon^{-1} N^{-1} f\left(\frac{\varepsilon}{x}\right) t_{d h}^{-1} \quad(1 \lesssim \beta \varepsilon),
$$

where $f$ is a function represented in Figure 1 by the appropriately labelled continuous line, and $t_{d h}$ is the dynamical time defined by Spitzer and Hart (1971a). Here we have taken $A=e^{-1}$ in (19) for convenience, while the dotted extension to the left shows 
the form that $f$ would take were distant encounters ignored entirely. Likewise the energy flux is

$$
\mathscr{E} \simeq 4.1 \frac{n}{n_{h}}\left\langle\beta^{-1}\right\rangle^{3 / 2} \beta^{1 / 2} N^{-1} g\left(\frac{\varepsilon}{x}\right) t_{d h}^{-1} \quad(1 \lesssim \beta \varepsilon),
$$

where the function $g$ is plotted also in Figure 1, the two curves having the same

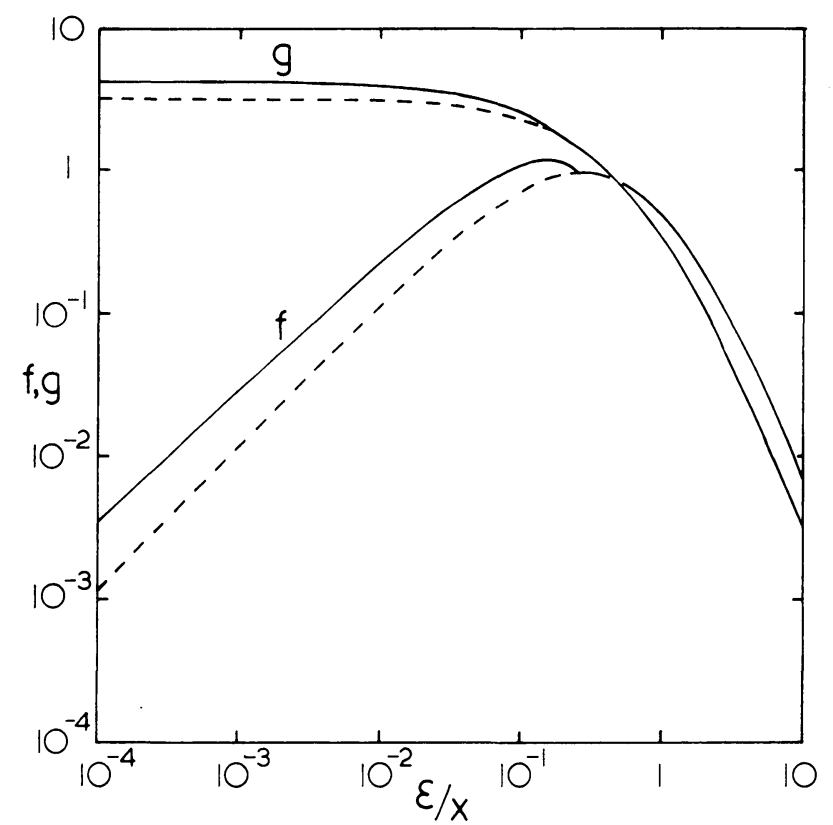

Fig. 1. Graphs of the functions appearing in Equations (26), (27) and (31), the argument being $\varepsilon / x$. The distinction between the continuous and dashed curves is explained in the text.

meaning as those for $f$. In this case, however, numerical integration is needed to obtain the results displayed. It is helpful to record Hénon's two-body escape rate (Hénon, 1969) in comparable notation, when it yields

and

$$
\mathscr{R}=0.011 t_{d h}^{-1}
$$

$$
\mathscr{E}=0.0050\left\langle\beta^{-1}\right\rangle t_{d h}^{-1} .
$$

In small systems containing at most a few hundred members it is clear that the mechanism of escape by encounters with a binary is competitive whenever a suitable binary is present. In large systems it is likely to be of importance only as soon as the core has evolved to the extent that the central density is high enough, although probably this also enhances the rate of two-body escape processes. Throughout the remainder of the evolution of the core the importance of the three-body process will persist, although the total mass lost during this phase need not be large because this part of the evolution is very brief. Furthermore, a single change in the internal binding 
energy of a pair which is sufficiently large to lead to the escape of a star from the cluster is probably also large enough to remove the binary at least into the halo, thus reducing the rate of further encounters with members of the core.

\section{Initial Binaries}

Too little is known about the process of star formation to rule out the possibility that a substantial fraction of stars are born as binaries, and yet enough is known to suggest strongly that it is likely (Larson, 1972). In this section we shall estimate the minimum abundance in which such 'initial binaries' would have to be present in order to exert a substantial influence on the evolution of the cluster.

Considering first soft binaries, we denote by $n(x) \mathrm{d} x$ the initial number-density within the energy interval $(x, x+\mathrm{d} x)$ in excess of what one would expect from the equilibrium distribution (4). These binaries would be destroyed on a time scale given by (10), and the rate at which their energy would be released is given by

$$
\dot{x} \simeq \frac{-1.2\left(0.6+\ln \frac{1}{\beta x}\right)}{\log (0.4 N)} \frac{n}{n_{h}}\left(\beta\left\langle\beta^{-1}\right\rangle\right)^{3 / 2} \beta^{-1} t_{r h}^{-1}
$$

(Heggie, 1974a), which is different from (9) because we here include those encounters which lead to the destruction of a binary. Hence the local rate per unit volume at which the binding energy of soft pairs would change is

$$
-\frac{1.2}{\log (0.4 N)} \int\left(0.6+\ln \frac{1}{\beta x}\right) \frac{n(x) \mathrm{d} x}{n} \frac{n}{n_{h}}\left(\beta\left\langle\beta^{-1}\right\rangle\right)^{3 / 2} n \beta^{-1} t_{r h}^{-1} .
$$

The result has been cast in this form to facilitate comparison with the rate at which energy is exchanged by collisional relaxation. This is of order $\frac{3}{2} n \beta^{-1} t_{r}^{-1}$, where $t_{r}$ is the local relaxation time, and using Chandrasekhar's expression (Chandrasekhar, 1942 , p. 201) we obtain the result

$$
1.1\left(\beta\left\langle\beta^{-1}\right\rangle\right)^{3 / 2} \frac{n}{n_{h}} n \beta^{-1} t_{r h}^{-1}
$$

From these formulae we see that the release of energy from an initial excess of soft pairs would have an influence on the evolution of the cluster comparable with that due to collisional relaxation only if their numbers were comparable with those of the single stars. Very soft pairs would be the most efficient, but the effect is a weak logarithmic one.

The release of binding energy by soft pairs would lead to a contraction of the cluster, but the mechanism would only persist for a time of order $t_{s}$, given by (10). Therefore the total effect of the binary process could be small, even though it might dominate for a short time, if most of the pairs were very soft and hence rapidly destroyed. An estimate of the total effect of their destruction can be obtained by 
computing their binding energy per unit volume, which is

$$
\int \beta x \frac{n(x) \mathrm{d} x}{n} n \beta^{-1} \text {. }
$$

Only if this were comparable with the local density of kinetic energy, i.e. $\frac{3}{2} n \beta^{-1}$, would the cumulative effect of initial soft pairs be important. The presence of the factor $\beta x$ implies that very soft pairs would be relatively inefficient.

The fact that the destruction of hard pairs may be neglected implies that the effects of possible initial hard binaries would not be confined to a brief early period within the first relaxation time. If, as a result of an encounter between a binary and a single star, the internal binding energy of the binary were to change by an amount $y$, then the binding energy of the single star with respect to the cluster would change by an amount $\Delta \varepsilon \simeq-\frac{2}{3} y$ (cf. the discussion of escape rates in the previous section). For a typical single star the rate at which such encounters would occur is

$$
\frac{3}{2} \int n(x) Q\left(x,-\frac{3}{2} \Delta \varepsilon\right) \mathrm{d} x,
$$

and so the mean rate at which the energy of single stars would change as a result of close encounters with hard pairs would be

$$
-\frac{0.50}{\log (0.4 N)} \int \frac{n(x) \mathrm{d} x}{n} \frac{n}{n_{h}}\left(\beta\left\langle\beta^{-1}\right\rangle\right)^{3 / 2} n \beta^{-1} t_{r h}^{-1}
$$

per unit volume, using (20). If $N=10^{5}$, this would be comparable with the effect of collisional relaxation only if the total number of hard pairs were as much as about ten times the number of single stars. We have neglected a sizable contribution to (30) from distant encounters, however.

If the number of binaries were really so large, other effects would require consideration. So far we have only considered 'superelastic' encounters between single stars and binaries, but relaxation would also occur as a result of 'elastic' encounters, in which binaries may be treated as point masses with twice the mass of the single stars. The presence of such a species would considerably decrease the two-body relaxation time, and a system consisting almost entirely of hard binaries would relax four times more quickly than one whose members were mostly single stars (cf. Chandrasekhar, 1942 , p. 201). Finally, if the proportion of binaries were large, the effect of non-elastic encounters between pairs would probably dominate that of superelastic encounters between pairs and single stars.

Since the effect of hard binaries would be to cause a decrease in the binding energy of the single stars, their existence initially in sufficiently large numbers would not only inhibit the formation in the system of a dense core of particles with high binding energy, but would also lead to enhanced loss of mass, as we shall see later. Furthermore, the consequent overall expansion of the system would much increase the efficiency of mass loss by tidal forces (Hayli, 1971), although the rate of occurrence of other dynamical processes would decrease as the mass-density decreased. 
There are two respects in which (30) is misleading, in addition to that pointed out by Dr. Hénon in the discussion of this paper. It implies, first, that the efficiency of hard pairs would be independent of their binding energy, $x$. This statement ignores the stochastic nature of encounters, for encounters with very hard pairs would typically yield very large changes of energy but these would occur very infrequently. Second, (30) obscures the fact that changes in energy due to encounters would not occur primarily as a succession of numerous small changes, but discretely. Thus only one change of order $-\varepsilon$ to the energy of each star, during the evolution of the system towards high central densities, would be sufficient to prevent formation of a core, even though the average rate of energy exchange by binaries could then be considerably smaller than that by collisional relaxation. To complement $(30)$ on all three criticisms, therefore, we shall calculate the expected number of times, $v$, that the energy of a single star suffers changes satisfying the inequality $\Delta \varepsilon<-\varepsilon_{0}$, where $\varepsilon_{0}$ is a positive constant, during a time interval $\tau t_{r h}$, where $\tau$ is a measure of the interval in units of $t_{r h}$. From (15) and (18), using the sorts of approximations employed in deriving the escape rate in Section 3 , we obtain

$$
v \simeq \frac{0.16 \tau}{\log (0.4 N)}\left(\beta\left\langle\beta^{-1}\right\rangle\right)^{3 / 2} \frac{n}{n_{h}} \frac{1}{\beta \varepsilon_{0}} \int \frac{n(x)}{n} f\left(\frac{\varepsilon_{0}}{x}\right) \mathrm{d} x \quad\left(\beta \varepsilon_{0} \gtrsim 1\right)
$$

where $f$ is the function plotted in Figure 1 .

Now we see that binaries with energies slightly larger than the required energy change are the most efficient. To estimate the rate of escape of stars from the system, we take for $\varepsilon_{0}$ the average change in energy required for escape, viz. $\frac{9}{2}\left\langle\beta^{-1}\right\rangle$ (Chandrasekhar, 1942, p. 206). Taking a uniform sphere with $N=10^{5}$ and $\tau=16$ we find that the coefficient of the integral is about 0.12 , and so it is again clear that only very large populations of hard binaries, now confined to a restricted range of energies between, say, $2 \beta^{-1}$ and $20 \beta^{-1}$, could lead to complete loss of single stars from the system during the time that would normally be required for complete collapse of the core. Clearly, considerably smaller populations could still be of importance in influencing the development of the core, especially when regard is paid to the facts that the logarithmic factor in (31) should really depend on the structure of the cluster as well as its total membership, and, a fortiori, that (31) depends on the spatial density, $n$.

From the discussion of Equation (29) it will be recalled that the population of hard pairs was required to be about ten times that of single stars in order that they should redistribute kinetic energy as efficiently as two-body relaxation. At first sight, therefore, it is curious that the rates of escape due to the two mechanisms are comparable when the number-density of hard binaries, with energies in the range of greatest efficiency, is as low as $0.02 n$, which we see by comparing (28) with the discussion of (31). The explanation is that the importance of small energy changes relative to large changes is much greater for two-body relaxation than for the binary mechanism; 
therefore a given population of binaries is much more efficient at producing escapers, to which process possibly only large changes in energy contribute, than at exchanging energy. Likewise, energy exchange by hard binaries is considerably enhanced relative to that by single stars if we consider the mean square energy change rather than the mean. Hence the minimum population of hard binaries needed to exert a significant influence on the evolution of the system would depend very much on the aspect under consideration.

\section{Outlook}

In the absence of a simple analytical theory for the evolution of stellar systems it is difficult to be more precise about the magnitude of the effect on the evolution of a system which the presence of a specified initial number of hard binaries would exert. Numerical experiments are needed in order to study this question in detail, and in this connexion the above calculation implies that pairs with a binding energy of about five or ten times the mean kinetic energy would be the most effective if included in an $N$-body computation or a Monte Carlo scheme.

Such studies would also establish the possible importance of three other effects which have a bearing on these questions. First, as mentioned above, from the point of view of two-body relaxation a hard binary may be regarded as a point mass with twice the mass of the single stars. Hence the process of mass segregation (e.g. Aarseth, 1973) might enhance the relative density of binaries near the centre of the system by over an order of magnitude, and so binaries may influence the evolution of the core even if their initial abundance is much less than the previous estimates of the minimum number required to have a significant effect on the development of the whole cluster. A second mechanism of a slightly different nature, but having a similar effect, comes into play as soon as energetic encounters between single stars and binaries begin to occur with significant frequency. If the internal energy of the binary changes by an amount $y$, then for the single star the change in binding energy relative to the cluster is $\Delta \varepsilon \simeq-\frac{2}{3} y$, and for the binary $\Delta \varepsilon \simeq-\frac{1}{3} y$. Since it is the binding energy per unit mass which discriminates core and halo members, we see that encounters in which significant changes occur in the internal energy of the binary are much more efficient at removing single stars from the core than binaries. Finally, in considering an abundance of binaries comparable with that of single stars, we repeat that the frequency of encounters between binaries may rival that of other types of encounter, and furthermore the cross-section for energetic encounters between two binaries almost certainly exceeds that for encounters between binaries and single stars.

The immediate difficulty in applying these results to real clusters is our ignorance of the dependence on the masses of the rate functions $Q$ in some important cases, although the following order-of-magnitude calculation suggests that the presence of different masses may not alter some of our conclusions. We consider encounters between a single star of mass $m_{3}$ and hard binaries of energy $x$, whose components have masses $m_{1}, m_{2}$. Let $n(x)$ be the number-density of such pairs. Then $\varepsilon$, the binding energy of the single star, changes as a result of (a) 'elastic' encounters, and (b) 'super- 
elastic' encounters. The effect of the first mechanism is to increase $\varepsilon$ at a rate of order

$$
\dot{\varepsilon} \sim n(x) m_{3} M_{12} M_{123} \frac{G^{2}}{\langle v\rangle}
$$

(Chandrasekhar, 1943), where $M_{12} \equiv m_{1}+m_{2}, M_{123} \equiv M_{12}+m_{3}$, and $\langle v\rangle$ is the mean particle speed in the system. Now let us consider the second mechanism. Taking a Keplerian approximation to the relative motion of the binary and the third body, the impact parameter required for an approach to a distance comparable with the semi-major axis of the binary is of order

$$
\frac{G}{\langle v\rangle}\left(\frac{m_{1} m_{2} M_{123}}{x}\right)^{1 / 2}
$$

If $m_{3} \ll m_{1}, m_{2}$ it is reasonable to suppose that the change in the velocity of the single star relative to the binary is approximately independent of $m_{3}$ and typically comparable with the relative velocity of its components, and so the average change in $\varepsilon$ is of order $\left(m_{3} M_{12}^{3} / m_{1} m_{2} M_{123}^{2}\right) x$. Hence the average rate of change due to inelastic encounters is of order

$$
\dot{\varepsilon} \sim-n(x) \frac{m_{3} M_{12}^{3}}{M_{123}} \frac{G^{2}}{\langle v\rangle},
$$

which has almost the same mass-dependence as (32) if $m_{3}$ is very small. The conclusion would then be that the relative importance of the two relaxation processes does not change significantly in the presence of different masses.

The observational position is also complicated by the existence of a spectrum of masses. Here the boundary between hard and soft pairs may be taken to lie at the energy of the most energetic single member of the cluster. If this is a star of mass $\mathscr{M}_{\max }$ say, and a velocity of $0.5 \mathrm{~km} \mathrm{~s}^{-1}$, typical of open clusters, the corresponding semi-major axis is

$$
a \simeq 4 \times 10^{3} \frac{\mathscr{M}_{1} \mathscr{M}_{2}}{\mathscr{M}_{\max }} \mathrm{AU}
$$

where $\mathscr{M}_{1}, \mathscr{M}_{2}$ are the masses of the components, all masses being expressed in solar units. With components exceeding the sun in mass, such binaries should be resolved visually at distances even as great as $1 \mathrm{kpc}$. In globular clusters, for which the coefficient in the corresponding expression would be smaller by a factor of about 100 , such binaries could not be detected by visual inspection.

Following the results of Section 2, binaries present initially with semi-major axes exceeding these limits would not be expected to persist for more than a relaxation time, at the most. In globulars, they would be difficult to detect, and should not have been expected to survive in the relaxed central regions of these objects. They should be sought in young open clusters less than about $10^{7}$ years old, and since our only interest is in an excess of very soft pairs over the numbers corresponding to an un- 
correlated pair distribution, they could be detected in relatively nearby clusters by considering the statistics of pair separations among cluster members, or by dividing the area of the cluster into squares and comparing the star counts with a Poisson distribution.

A search for hard pairs, especially those with semi-major axes between about 0.05 and 0.5 times the value in (33), could usefully be conducted in the central regions of open clusters of all ages. They would be detectable by direct visual techniques only in the most nearby systems, but in the remainder only interferometric methods and, in special cases, occultation photometry would be feasible. Spectroscopy does not afford much prospect of success except in globular clusters.

The other source of information on the abundance of binaries is the solar neighbourhood, although the extent of its relevance to the stellar population in clusters is unknown. Considering the twelve known pairs within 5 pc (van de Kamp, 1971) having semi-major axes between 1 and $100 \mathrm{AU}$, we find that $n^{-1} \int n(x) \mathrm{d} x \simeq 0.4$. The masses cover a wide range exceeding a decade, but the average is about $0.6 \mathscr{M}_{\odot}$, and so these binaries, were they situated in an open or globular cluster, would roughly include those values of the binding energy which are most effective in influencing the evolution of the system. Furthermore, there is no guarantee from the conclusions of Section 4 that an abundance of this order would not have a significant effect on the development of a core, and it would certainly enhance substantially the rate of escape from the cluster as a whole.

\section{Acknowledgements}

I am grateful to many people for numerous discussions on binary problems, but especially to Dr S. J. Aarseth, who suggested to me some years ago that the topic was ready for detailed study, and who has followed the progress of the work with deep interest. I am happy to acknowledge in addition the financial support of the U.K. Science Research Council and of Trinity College, Cambridge, as well as the hospitality of the former Institute of Theoretical Astronomy, and now of the Institute of Astronomy, Cambridge.

\section{References}

Aarseth, S. J.: 1971a, Astrophys. Space Sci. 13, 324.

Aarseth, S. J.: 1971b, Astrophys. Space Sci. 14, 20.

Aarseth, S. J.: 1973, Vistas in Astronomy 15, 13.

Aarseth, S. J.: 1974, Astron. Astrophys. 35, 237.

Aarseth, S. J., Hénon, M., and Wielen, R.: 1974, Astron. Astrophys. 37, 183.

Aarseth, S. J. and Hills, J. G.: 1972, Astron. Astrophys. 21, 255.

Anosova, Zh. P.: 1969, Astrophysics 5, 81.

Chandrasekhar, S.: 1942, Principles of Stellar Dynamics, University of Chicago Press, Chicago, Ill.

Chandrasekhar, S.: 1943, Astrophys. J. 97, 255.

Gurevich, L. E. and Levin, B. Yu.: 1950, Astron. Zh. 27, 273, also NASA TT F-11,541 (1968).

Hayli, A.: 1971, Astrophys. Space Sci. 13, 309.

Heggie, D. C. : 1974a, 'Binary Evolution in Stellar Dynamics', to be submitted.

Heggie, D. C.: 1974b, in Y. Kozai (ed.). 'The Stability of the Solar System and of Small Stellar Systems', IAU Symp. 62, 225. 
Hénon, M.: 1969, Astron. Astrophys. 2, 151.

Hénon, M.: 1971, Astrophys. Space Sci. 13, 284.

King, I. R.: 1958, Astron. J. 63, 114.

Larson, R. B. : 1970a, Monthly Notices Roy. Astron. Soc. 147, 323

Larson, R. B.: 1970b, Monthly Notices Roy. Astron. Soc. 150, 93.

Larson, R. B. : 1972, Monthly Notices Roy. Astron. Soc. 156, 437.

Miller, R. H.: 1975, this volume, p. 65.

Miller, R. H. and Parker, E. N.: 1964, Astrophys. J. 140, 50.

Ross, J., Light, J. C., and Schuler, K. E.: 1969, in A. R. Hochstim (ed.), Kinetic Processes in Gases and Plasmas, Academic Press, New York and London, p. 281.

Spitzer, L., Jr and Hart, M. H.: 1971a, Astrophys. J. 164, 399.

Spitzer, L., Jr and Hart, M. H.: 1971b, Astrophys. J. 166, 483.

Spitzer, L., Jr and Saslaw, W. C.: 1966, Astrophys. J. 143, 400.

Szebehely, V.: 1972, Celes. Mech. 6, 84.

Valtonen, M. J.: 1974, in Y. Kozai (ed.), 'The Stability of the Solar System and of Small Stellar Systems', IAU Symp. 62, 211

van Albada, T. S. : 1968, Bull. Astron. Inst. Neth. 19, 479.

van de Kamp, P.: 1971, Ann. Rev. Astron. Astrophys. 9, 103.

von Hoerner, S.: 1958, Z. Astrophys. 44, 221.

von Hoerner, S. : 1963, Z. Astrophys. 57, 47.

von Hoerner, S. : 1968, Bull. Astron., 3ème Série 3, 147.

\section{DISCUSSION}

Lynden-Bell: I would like to ask a strange question : is the average total mass of a binary pair greater or less than the average mass of single stars? The point is that if a gas cloud either forms a pair or a single star there might be no reason why binaries should be heavier and should sink to the middle of a cluster.

Heggie: The general question here is how does the abundance of binaries depend on the masses of the components? Your remark would imply that it is small for the highest masses, but observational evidence should also be considered. Prof. Spitzer has pointed out privately that since stellar evolution removes the largest masses first, after a time the masses of binary components could be as large as those of single stars even if your idea is correct.

Hayli: How much are binaries responsible for the disruption of soft binaries?

Heggie: Hard binaries act on soft binaries approximately as single stars with a mass equal to the combined mass of their components. Heavy stars are more effective at disruption than light ones, but the relative effect of binaries on the disruption of soft pairs is chiefly determined by the abundance of hard binaries relative to single stars.

Hénon: Is not it true that a large fraction of the energy given away by the hard binaries is carried out of the cluster by the escaping stars? In this case, the evolution of the cluster would be less affected than your computation indicates.

Heggie: It is true that mass lost from the system has a smaller effect on the evolution of the core than mass which is transferred from the core to the halo. This is another reason why it is better to calculate the number of energy changes rather than the rate at which energy is transferred. Then if we consider the binaries whose energies lie in the range of greatest efficiency, we see from the expression for $v$. that the number of energy changes leading to escape is considerably less than that which leads to transfer from core to halo. This is not true for extremely hard pairs, and so the effect of your remark is, again, to exclude such pairs from consideration.

Lecar: What happens to binaries with $\beta x \approx 1$ ?

Heggie: Obviously there is some value of $\beta x$, about one, where $\langle\dot{x}\rangle=0$, but I have not calculated what it is. As shown in the paper, binaries which are only slightly more energetic than this are very unlikely to be disrupted. It is more difficult to discuss with any precision the behaviour of soft pairs in some range less than this value, which leads, for example, to considerable uncertainty in the rate at which hard pairs form from soft ones. More work is required here.

Lecar: Why do 'soft' binaries become softer and 'hard' binaries become harder?

Heggie: Gurevich and Levin remarked that the internal kinetic energy of a very soft pair is typically much less than the kinetic energy in the relative motion of the binary and a third body, whence equipartition leads to increased internal kinetic energy in the binary, i.e. to disruption. If a third body closely ap- 
proaches a very hard binary of energy $x$, such that the energy of relative motion is of order $\beta^{-1} \ll x$, two things may happen: either $x$ increases, when the third body can escape again; or else $x$ decreases. In this case, since the relative velocity of the binary and the third body at the time of encounter is in general almost independent of its initial value, the change in energy must be of order $-x$, thus binding the third body to the binary. When the resulting triple system disrupts, the third body will escape with energy of order $x$, and so in this case also the net change in the binding energy of the binary is positive.

Spitzer: It would certainly be interesting to incorporate these binary star effects in the Monte Carlo calculations. An important process is likely to be collision between pairs of binaries, since as you have suggested the number density of binaries may exceed that of single stars, at least in the cluster core. Will your paper include cross-sections and similar data for these encounters between two binary systems?

Heggie: No, although it ought to be possible to estimate such results, from the data on encounters between binaries and single stars, if the energies of the two pairs are very different. Numerical computations are probably necessary when this condition is not met.

Lecar: What is the distribution of eccentricities of hard binaries?

Heggie: Numerical evidence shows that the distribution is well relaxed, i.e. $f(e)=2 e$. Analysis of the effects of encounters indeed indicates that eccentricity relaxes faster than binding energy as a result of "distant" encounters. 